Type 2 diabetes and sepsis outcome

\title{
Trajectory of Type 2 Diabetes in Sepsis Outcome: Impacts of Diabetic Complication Burdens, Initial Glucose Level, and HbA1c: Population-Based Cohort Study Combining with Nationwide and Hospital-Based Database
}

Running title: Type 2 diabetes and sepsis outcome

Authors: Ming-Shun Hsieh, MD ${ }^{1-4}$, Sung-Yuan Hu, MD, MS ${ }^{5}$, Chorng-Kuang How, MD, PhD ${ }^{3,4}$, Yi-Tzu Lee, $\mathrm{MD}, \mathrm{PhD}^{3,4}$, Chen-June Seak, MD, MS ${ }^{6}$, Vivian Chia-Rong Hsieh, $\mathrm{PhD}^{7}$, Jen-Huai Chiang, $\mathrm{MS}^{8}$, Chiann-Yi Hsu, MS ${ }^{9}$, Sheng-Hsiang Ma, MD, Pau-Chung Chen, MD, PhD ${ }^{1,10 *}$, and Health Data Analysis in Taiwan (hDATa) Research Group

\section{Affiliations:}

${ }^{1}$ Institute of Occupational Medicine and Industrial Hygiene, National Taiwan University College of Public Health, Taipei, Taiwan;

${ }^{2}$ Department of Emergency Medicine, Taipei Veterans General Hospital, Taoyuan Branch, Taoyuan, Taiwan;

${ }^{3}$ Department of Emergency Medicine, Taipei Veterans General Hospital, Taipei, Taiwan;

${ }^{4}$ School of Medicine, National Yang-Ming University, Taipei, Taiwan;

${ }^{5}$ Department of Emergency Medicine, Taichung Veterans General Hospital, Taichung, Taiwan;

${ }^{6}$ Department of Emergency Medicine, Lin-Kou Medical Center, Chang Gung Memorial Hospital, Taoyuan, Taiwan;

${ }^{7}$ Department of Health Services Administration, China Medical University, Taichung, Taiwan;

${ }^{8}$ Management Office for Health Data, China Medical University, Taichung, Taiwan;

${ }^{9}$ Department of Medical Research, Taichung Veterans General Hospital, Taichung, Taiwan;

${ }^{10}$ Department of Environmental and Occupational Medicine, National Taiwan University College of Medicine and Hospital, Taipei, Taiwan;

*Correspondence to Pau-Chung Chen, MD, PhD, (for all communication) Institute of Occupational Medicine and Industrial Hygiene, National Taiwan University College of Public Health, Taipei, Taiwan;

E-mail: pchen@ntu.edu.tw

Keywords: Diabetes mellitus, sepsis, diabetes complication severity index score;

Total word count: 2691 
Type 2 diabetes and sepsis outcome

\section{Author/co-authors contact details}

Ming-Shun Hsieh, $\mathrm{MD}^{1-4}$, edmingshun@gmail.com

Sung-Yuan Hu, MD, MS55, song9168@pie.com.tw

Chorng-Kuang How, MD, PhD ${ }^{3,4}$, ckhow@vghtpe.gov.tw

Yi-Tzu Lee, MD, PhD ${ }^{3,4}$, yitzulee@gmail.com

Chen-June Seak, MD, MS ${ }^{6}$, julianseak@hotmail.com

Vivian Chia-Rong Hsieh, PhD ${ }^{7}$, hsiehchiarong@gmail.com

Jen-Huai Chiang, MS , zinvii@gmail.com

Chiann-Yi Hsu, MS9, chiannyi314@gmail.com

Sheng-Hsiang Ma, MD4, almighty914@gmail.com

Pau-Chung Chen, MD, PhD ${ }^{1,10 *}$, pchen@ntu.edu.tw 
Type 2 diabetes and sepsis outcome

1 Abstract

2 Background

3 Diabetic patients have an increased risk of infections; however, the association between type 2

4 diabetes and hospital outcomes of sepsis remains controversial when the diabetes severity is not 5 considered. We examined this association using nationwide and hospital-based databases 6 concomitantly.

\section{Methods}

8 The first part of this study was conducted using 2 nationwide databases: the Longitudinal Cohort of Diabetes Patients and the Longitudinal Health Insurance Database 2000. The diabetic complication burden was evaluated using the adapted Diabetes Complications Severity Index score (aDCSI score). In the second part, we used the hospital-based database with laboratory data, such as initial blood glucose and $\mathrm{HbA} 1 \mathrm{c}$ levels, to make comparisons between surviving and dead patients with type 2 diabetes and sepsis.

\section{Results}

The nationwide study included 19,719 type 2 diabetic sepsis patients and an equal number of non-diabetic patients. The diabetic sepsis patients had an increased odds ratio (OR) of 1.14 (95\% $\mathrm{Cl}$ 1.1-1.19) for hospital mortality. The OR for mortality increased as the complication burden increased (diabetic sepsis patients with aDCSI scores of $0,1,2,3,4$, and $\geq 5$ had ORs of $0.91,0.87$, $1.14,1.25,1.56$, and 1.77 for mortality, respectively (all $P<0.001$ and $P$ for trend $<0.001$ )).

A total of 1,054 diabetic sepsis patients were included from the hospital-based database. Initial blood glucose levels in the surviving and dead diabetic sepsis patients did not differ significantly: $273.9 \pm 180.3$ versus $266.1 \pm 200.2(\mathrm{mg} / \mathrm{dL})(P=0.095)$. Moreover, the surviving diabetic sepsis patients did not have a lower HbA1c $(\%): 8.4 \pm 2.6$ versus $8.0 \pm 2.5(P=0.078)$.

\section{Conclusions}

In the case of type 2 diabetic sepsis patients, the diabetes-related complication burden is the major determinant of hospital mortality rather than the diabetes itself. Contrary to popular belief, initial blood glucose and $\mathrm{HbA1c}$ levels may not be as important as previously thought. 
Type 2 diabetes and sepsis outcome

\section{Introduction}

Sepsis is a leading cause of mortality in critical care worldwide.[1-3] In addition to mortality, sepsis may also induce acute organ dysfunction, and even cause long-term post-sepsis cardiovascular diseases.[4] The reported incidence of sepsis varies; however, it presents an undoubtedly increasing trend that is reflected in the aging population and greater recognition of this condition. Treating sepsis patients causes significant national financial burdens.

Diabetes is an important comorbid condition in sepsis because of its high prevalence.[5] It has generally been believed that diabetic patients are more prone to infections than the general population.[6] However, the influence of diabetes on the outcome of sepsis remains inconclusive. Higher mortality rates in the patients with diabetes were reported[7-12]; however, some others found no influence[13-16], and even protective effects of diabetes in sepsis.[17-20]

The most frequently proposed study limitations of this debate were the study designs: the epidemiological studies using large cohorts can avoid the selection bias that is frequently observed in hospital-based studies, however, detailed clinical information is usually not available. Most importantly, many studies failed to consider the influence of diabetic complication severity.

$\mathrm{HbA1c}$ is commonly used to measure blood glucose control in diabetic patients and has also been proposed as an independent predictor for hospital mortality in sepsis patients.[21] However, its importance in diabetic sepsis patients requires further study due to limited information. Hyperglycemia was shown to impair polymorphonuclear neutrophil function and cytokine production. However, it was reported that high initial glucose levels were not associated with increased mortality in the diabetic sepsis patients.[22] Furthermore, tight glucose control did not seem to be significantly associated with reduced hospital mortality in critical patients.[23, 24] The influence of $\mathrm{HbA1C}$ and initial glucose levels on the outcome of sepsis deserves further investigation.

In the current study, using the representative nationwide database and the hospital-based database from multi-centers with laboratory data, we examined the association between type 2 diabetes and sepsis outcomes, and specifically focused on (1) whether diabetes itself increases the risk of mortality in hospitalized sepsis patients or depends on the diabetes complication burdens and (2) whether initial blood glucose levels and $\mathrm{HbA1c}$ affect the hospital course and outcomes. 
Type 2 diabetes and sepsis outcome

\section{Methods}

\section{Data sources and study participants}

\section{Nationwide database}

In the first part of this study, we conducted a nationwide cohort study using data from the National Health Insurance Research Database (NHIRD). The diagnosis codes of the International Classification of Diseases, Ninth Revision, Clinical Modification (ICD-9-CM) are used in the NHIRD to identify specific diagnoses. Sepsis patients were retrieved using the ICD-9-CM codes 038. The specificity of a sepsis diagnosis in the NHIRD has been validated in previous studies.[25] The infection site classification was performed similar to Angus et al. (Supplement Table 1).[26]

The patients were classified as using certain drugs if they took them for more than one month within a one-year period prior to the index hospitalization. The index date was defined as the first date of index hospitalization. The drug codes are shown in Supplement Table 2. The procedures during hospitalization were defined by using the claims data (Supplement Table 3).

Initially, we used the Longitudinal Cohort of Diabetes Patients (LHDB) of the NHIRD, which contains randomized selected data $(120,000$ patients / year) from patients with newly diagnosed diabetes to retrieve the study cohort of type 2 diabetic first episode sepsis patients.[27] The patients in the study cohort should have been diagnosed to have type 2 diabetes prior to the index hospitalization to allow for the evaluation of diabetic complication status by using the adapted Diabetes Complications Severity Index score (aDCSI score) (Supplement Table 4).[28, 29] (The original form of DCSI score had some missing and we had corrected in the supplement table)

The Diabetes Complications Severity Index (DCSI) was first developed by Young et al.[28] The DCSI is a useful tool to adjust for the baseline severity of diabetic complications and to predict hospitalization and mortality. The aDCSI score was modified from the DCSI score and had been validated in the NHIRD.[30] The aDCSI score included seven categories of complications: cardiovascular disease, nephropathy, neuropathy, retinopathy, peripheral vascular disease, stroke, and metabolic emergency events.

The comparison cohort, which was composed of non-diabetic first episode sepsis patients, was retrieved from the Longitudinal Health Insurance Database 2000 (LHID2000). The LHID2000 used in this study contains the medical information of 1 million beneficiaries, randomly sampled from the registry of all beneficiaries in 2000 . This study was approved by the Institutional Review Board of China Medical University (CMUH104-REC2-115). 
Type 2 diabetes and sepsis outcome

\section{Hospital-based database}

In the second part of this study, we retrieved data on the type 2 diabetic first episode sepsis patients between 2006 and 2012 from the electronic databases of three medical centers. Most of these diabetic sepsis patients were included in the LHDB. Laboratory data, including initial blood glucose level, $\mathrm{HbA1c}$, and initial lactate level; hospital outcomes, including ICU (intensive care unit) admission, hospital and 28-day mortalities; the received procedures (including mechanical ventilation and emergent hemodialysis); blood culture results were collected for further analysis. This study was approved by the Institutional Review Board of Taichung Veterans General Hospital (No. CE13233).

The participant selection process of the nationwide and hospital-based databases was showed in Supplement Figure 1 and Supplement Figure 2. Only type 2 diabetic patients were included for further study. In the hospital-based database, the initial blood glucose level was obtained on the admission day ether in the emergency department or ward before receiving any acute glucose-lowering injection therapy, that is, insulin; the HbA1c level should be obtained in a period of three days around the admission day.

\section{Statistical analyses}

In the hospital-based database, the type 2 diabetic and non-diabetic sepsis patients were matched by age and gender. In the nationwide database, the study cohort from the LHDB and the comparison cohort from the LHID2000 were matched by the propensity score matching method. For each patient, we calculated the propensity score using the multivariate logistic regression by entering age, gender, income, urbanization level, hospital level, baseline comorbidities, and infection site.

Differences in demographic characteristics, comorbidities, medications, and laboratory data were examined using Chi-square, Mann-Whitney and two-sample t-tests. Odds ratios (ORs) with 95\% confidence intervals (95\% Cls) were calculated in the logistic regression model. The Kaplan-Meier analysis with log-rank test was conducted to compare the hospital outcome in patients with different initial blood glucose levels and HbA1c. The statistical analyses were performed using SAS 9.4 statistical package (SAS Institute Inc., Cary, NC, USA). A P value of 0.05 was considered significant. 
Type 2 diabetes and sepsis outcome

\section{Sensitivity analysis}

2 Because of limited cases with the data of $\mathrm{HbA1C}$ using the strict criteria of three days around the 3 admission date, we further conducted a sensitivity analysis. The HbA1c data was re-collected with 4 a wider time period of one month prior to the admission date to re-examine the effect of HbA1c. 
Type 2 diabetes and sepsis outcome

1 Results

First part: nationwide database

From the LHDB and LHID2000 between 1999 and 2012, after propensity score matching, 19,719 type 2 diabetic and an equal number of non-diabetic first episode sepsis patients were retrieved as the study and comparison cohorts. The demographic characteristics, comorbidities, medications, infection sites, and received procedures of the study and comparison cohorts were showed in Supplement Table 5.

Before matching, the diabetic sepsis patients had a higher prevalence of genitourinary tract infection (33.65\% versus $27.71 \%$ ) and soft tissue/musculoskeletal system infection related sepsis (5.77\% versus $4.54 \%$ ) (both $P<0.0001$ ). Also, the diabetic sepsis patients more frequently received respiratory support (mechanical ventilation: $39.42 \%$ versus $38.18 \%$; non-invasive positive pressure ventilation: $7.12 \%$ versus $6.75 \%$, both $P<0.0001)$ and emergent dialysis $(11.56 \%$ versus $8.28 \%$, $P<0.0001$, respectively).

After matching, in the further multivariate analysis, type 2 diabetic sepsis patients had an increased OR of 1.14 (95\% confidence level $[\mathrm{Cl}] 1.1-1.19, P<0.0001)$ for mortality after adjusting for age, gender, insurance premium (as a proxy for household income), urbanization level, and hospital level (Supplement Table 6).

In the analysis according to diabetic complication burdens, the patients with aDCSI scores of 0,1 , 2, 3, 4, and $\geq 5$ had ORs of $0.91(95 \% \mathrm{Cl}, 0.85-0.97), 0.87(95 \% \mathrm{Cl}, 0.8-0.96), 1.14(95 \% \mathrm{Cl}$, 1.07-1.22), 1.25 (95\% Cl, 1.13-1.38), 1.56 (95\% Cl, 1.43-1.7), and 1.77 (95\% Cl, 1.61-1.96) for mortality during sepsis, respectively (all $P<0.001$ and $P$ for trend $<0.001$ ) (Figure 1 ). In the subgroup analysis stratified by age (every 10 years), the type 2 diabetic sepsis patients with higher aDCSI scores had increased ORs for mortality compared to those with lower scores, particularly in the 30-39 age group (Supplement Figure 3). 
Type 2 diabetes and sepsis outcome

1 Figure 1 Logistic regression analysis analyzing the odds ratios of hospital mortality in sepsis 2 patients with type 2 diabetes and different diabetic complication burdens (aDCSI scores).

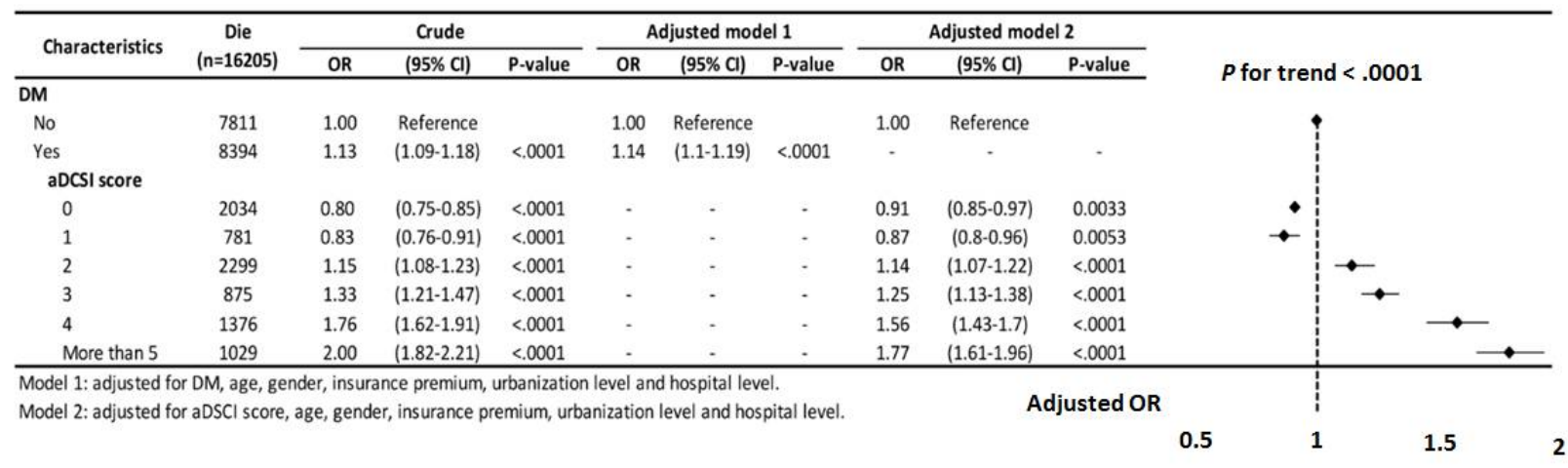

\section{Second part: hospital-based database}

5 From the hospital-based database, we initially included 4,984 sepsis patients between 2006 and 6 2012. After matching for age and gender, there were 1,054 type 2 diabetic and 2,108 non-diabetic 7 sepsis patients included for further analysis. The type 2 diabetic sepsis patients had a higher 8 prevalence of receiving hemodialysis $(23.2 \%$ versus $16.9 \%, P<0.001)$ during hospitalization (Table 9 1). The type 2 diabetic sepsis patients had higher hospital mortality rate $(45.2 \%$ versus $42.3 \%$, $10 P=0.138)$ and 28 -day mortality rate (35.5\% versus $32.8 \%, P=0.147)$ compared to the non-diabetic 11 sepsis patients. The type 2 diabetic sepsis patients had higher prevalence of gram-positive coccus 12 bacteremia ( $16.8 \%$ versus $14.4 \%, P=0.089$ ) but lower prevalence of gram-negative bacillus 13 bacteremia ( $19.1 \%$ versus $20.7 \%, P=0.294$ ) compared to the non-diabetic sepsis patients, although 14 the $P$ value did not reach the significance. 
Type 2 diabetes and sepsis outcome

Table 1 Demographic characteristics, comorbidities, laboratory data, hospital course, and outcomes of matched type 2 diabetic and non-diabetic sepsis patients

\begin{tabular}{|c|c|c|c|c|}
\hline \multirow{2}{*}{ Variables } & \multirow{2}{*}{ Total $(n=3162)$} & \multicolumn{2}{|c|}{ DM } & \multirow{2}{*}{$P$ value } \\
\hline & & Yes $(n=1054)$ & No $(n=2108)$ & \\
\hline Age & $70.4 \pm 13.1$ & $70.3 \pm 12.9$ & $70.4 \pm 13.1$ & 0.779 \\
\hline Male & 1956(61.9) & 652(61.9) & 1304(61.9) & 1.000 \\
\hline Septic shock & $1867(59.0)$ & $616(58.4)$ & $1251(59.3)$ & 0.655 \\
\hline Hospital mortality & 1368(43.3) & $476(45.2)$ & $892(42.3)$ & 0.138 \\
\hline 28-day mortality & 1066(33.7) & $374(35.5)$ & $692(32.8)$ & 0.147 \\
\hline Hemodialysis & $602(19.0)$ & $245(23.2)$ & $357(16.9)$ & $<0.001$ \\
\hline Mechanical ventilation & 1897(60.0) & $658(62.4)$ & $1239(58.8)$ & 0.053 \\
\hline ICU admission & 1771(56.0) & $606(57.5)$ & 1165(55.3) & 0.249 \\
\hline Length of ICU stay & $15.6 \pm 14.3$ & $14.6 \pm 13.8$ & $16.0 \pm 14.6$ & 0.020 \\
\hline \multicolumn{5}{|l|}{ APACH II score } \\
\hline$(n=557 \text { vs } 1063)^{\pi}$ & $25.0 \pm 7.0$ & $25.3 \pm 7.1$ & $24.9 \pm 7.0$ & 0.292 \\
\hline Length of hospital stay" & $23.5 \pm 25.5$ & $23.0 \pm 27.5$ & $23.7 \pm 24.4$ & 0.214 \\
\hline \multicolumn{5}{|l|}{ Comorbidities } \\
\hline HTN & $931(29.4)$ & $463(43.9)$ & $468(22.2)$ & $<0.001$ \\
\hline Hyperlipidemia & $54(1.7)$ & $36(3.4)$ & $18(0.9)$ & $<0.001$ \\
\hline COPD & $287(9.1)$ & $72(6.8)$ & $215(10.2)$ & 0.002 \\
\hline CLD & $244(7.7)$ & $81(7.7)$ & $163(7.7)$ & 1.000 \\
\hline CKD & 1019(32.2) & $410(38.9)$ & $609(28.9)$ & $<0.001$ \\
\hline PAOD & $80(2.5)$ & $43(4.1)$ & $37(1.8)$ & $<0.001$ \\
\hline IHD & $124(3.9)$ & $55(5.2)$ & $69(3.3)$ & 0.010 \\
\hline Cancer & $958(30.3)$ & $226(21.4)$ & $732(34.7)$ & $<0.001$ \\
\hline Stroke & $273(8.6)$ & $120(11.4)$ & $153(7.3)$ & $<0.001$ \\
\hline $\mathrm{CCl}$ score ${ }^{\pi}$ & $3.4 \pm 2.7$ & $3.7 \pm 2.4$ & $3.2 \pm 2.8$ & $<0.001$ \\
\hline \multicolumn{5}{|l|}{ Bacterial cultures } \\
\hline GPC & $481(15.2)$ & $177(16.8)$ & 304 (14.4) & 0.089 \\
\hline GNB & $638(20.2)$ & $201(19.1)$ & $437(20.7)$ & 0.294 \\
\hline \multicolumn{5}{|l|}{ Laboratory data } \\
\hline Glucose & $191.4 \pm 141.8$ & $270.4 \pm 189.4$ & $149.1 \pm 80.8$ & $<0.001$ \\
\hline WBC $\left(x 10^{3}\right)$ & $13.2 \pm 13.5$ & $14.2 \pm 12.1$ & $12.7 \pm 14.1$ & $<0.001$ \\
\hline $\mathrm{Hb}$ & $12.0 \pm 2.7$ & $12.2 \pm 2.6$ & $12.0 \pm 2.7$ & 0.057 \\
\hline $\operatorname{PLT}\left(x 10^{6}\right)$ & $1.9 \pm 1.3$ & $2.1 \pm 1.6$ & $1.9 \pm 1.2$ & $<0.001$ \\
\hline $\mathrm{Cr}$ & $2.1 \pm 1.9$ & $2.4 \pm 2.1$ & $1.9 \pm 1.8$ & $<0.001$ \\
\hline Bilirubin & $0.9 \pm 2.0$ & $0.8 \pm 1.9$ & $0.9 \pm 2.0$ & $<0.001$ \\
\hline Lactate & $31.7 \pm 31.3$ & $32.9 \pm 34.0$ & $31.0 \pm 29.7$ & 0.259 \\
\hline
\end{tabular}

Chi-Square test. "Mann-Whitney test. Continuous data were expressed as mean \pm SD. Categorical data were expressed as number (percentage). 
Type 2 diabetes and sepsis outcome

1 Acute physiologic and chronic health (APACH), Charlson comorbidity index (CCl), Chronic kidney 2 disease (CKD), Chronic liver disease (CLD), Chronic obstructive pulmonary disease (COPD), 3 Gram-negative bacillus (GNB), Gram-positive coccus (GPC), Hypertension (HTN), Intensive care unit (ICU), Ischemic heart disease (IHD), Peripheral arterial occlusion disease (PAOD)

In the univariate and further multivariate logistic regression analyses, type 2 diabetes was associated with an increased risk of hospital mortality during the sepsis course (adjusted OR=1.31, $95 \% \mathrm{Cl}, 1.11-1.54, P=0.002$ ) (Supplement Table 7). But the Kaplan-Meier analysis with log-rank test did not show a significant difference of hospital course of mortality between the type 2 diabetic and non-diabetic sepsis patients $(P=0.122)$ (Figure $2 A)$.

The 1,054 type 2 diabetic sepsis patients were divided into surviving and dead groups for further comparison (Supplement Table 8). Initial blood glucose levels in the surviving and dead diabetic sepsis patients did not differ significantly: $273.9 \pm 180.3$ versus $266.1 \pm 200.2$ (mg/dL) $(P=0.095)$ (Figure 2B). Furthermore, the surviving diabetic sepsis patients did not have lower HbA1c (\%) than the dead diabetic sepsis patients: $8.4 \pm 2.6$ versus $8.0 \pm 2.5(P=0.078)$. In the further logistic regression analysis, the univariate analysis which included age, gender, $\mathrm{CCl}$ score and important laboratory data, showed an OR of $1.00(95 \% \mathrm{Cl}, 1.00-1.00, P=0.532)$ for initial glucose levels and $0.94(95 \% \mathrm{Cl}, 0.86-1.02, P=0.143)$ for HbA1c (Supplement Table 9). The Kaplan-Meier analysis with log-rank test also showed that the hospital mortality during the sepsis course did not differ between type 2 diabetic sepsis patients with different initial blood glucose levels ( $\leq 200,201-400$, and $>400 \mathrm{mg} / \mathrm{dL}$ ) and HbA1c ( $\leq 7$ and $>7 \%$ ) (Figure 3 ) (Supplement Table 10 and Supplement Table 11).

Figure 2A The Kaplan-Meier analysis with log-rank test for hospital course of mortality between the type 2 diabetic and non-diabetic sepsis patients. Figure 2B Comparison of Initial blood glucose levels in the surviving and dead diabetic sepsis patients. 
Type 2 diabetes and sepsis outcome

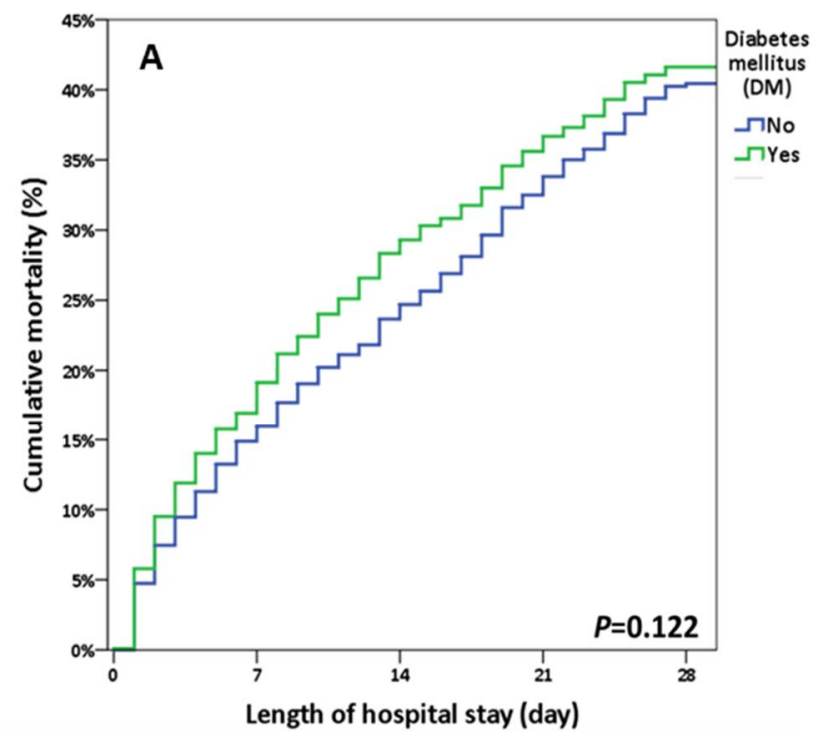

No. at risk

$\begin{array}{rrrrrrr} & \text { No } & 2108 & 1595 & 1189 & 826 & 591 \\ 1 & \text { Yes } & 1052 & 791 & 560 & 401 & 284\end{array}$

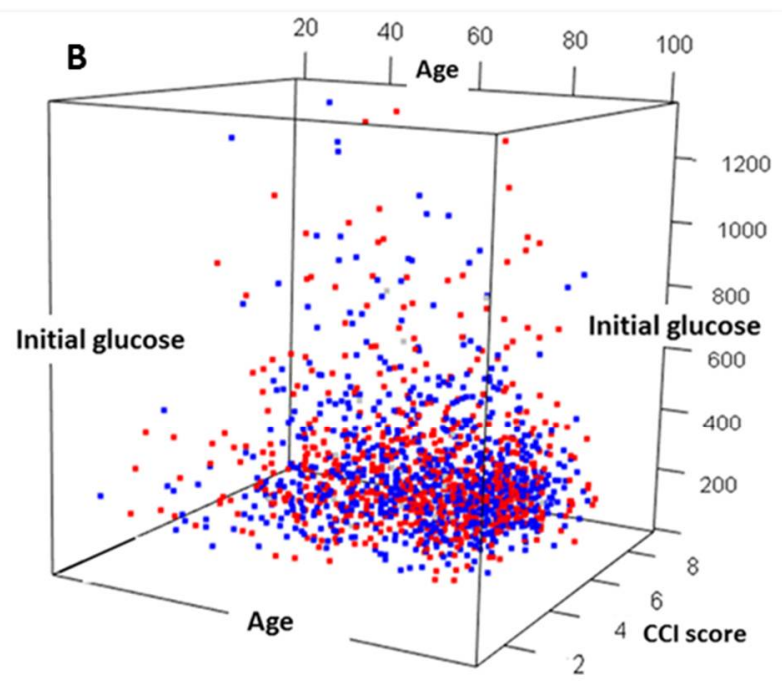

- Survived diabetic sepsis patients Initial glucose $273.9 \pm 180.3 \mathrm{mg} / \mathrm{dL}$

- Expired diabetic sepsis patients Initial glucose $266.1 \pm 200.2 \mathrm{mg} / \mathrm{dL}$

4 Figure 3 Kaplan-Meier analysis with log-rank test for type 2 diabetic sepsis patients with different 5 initial blood glucose levels and HbA1c.
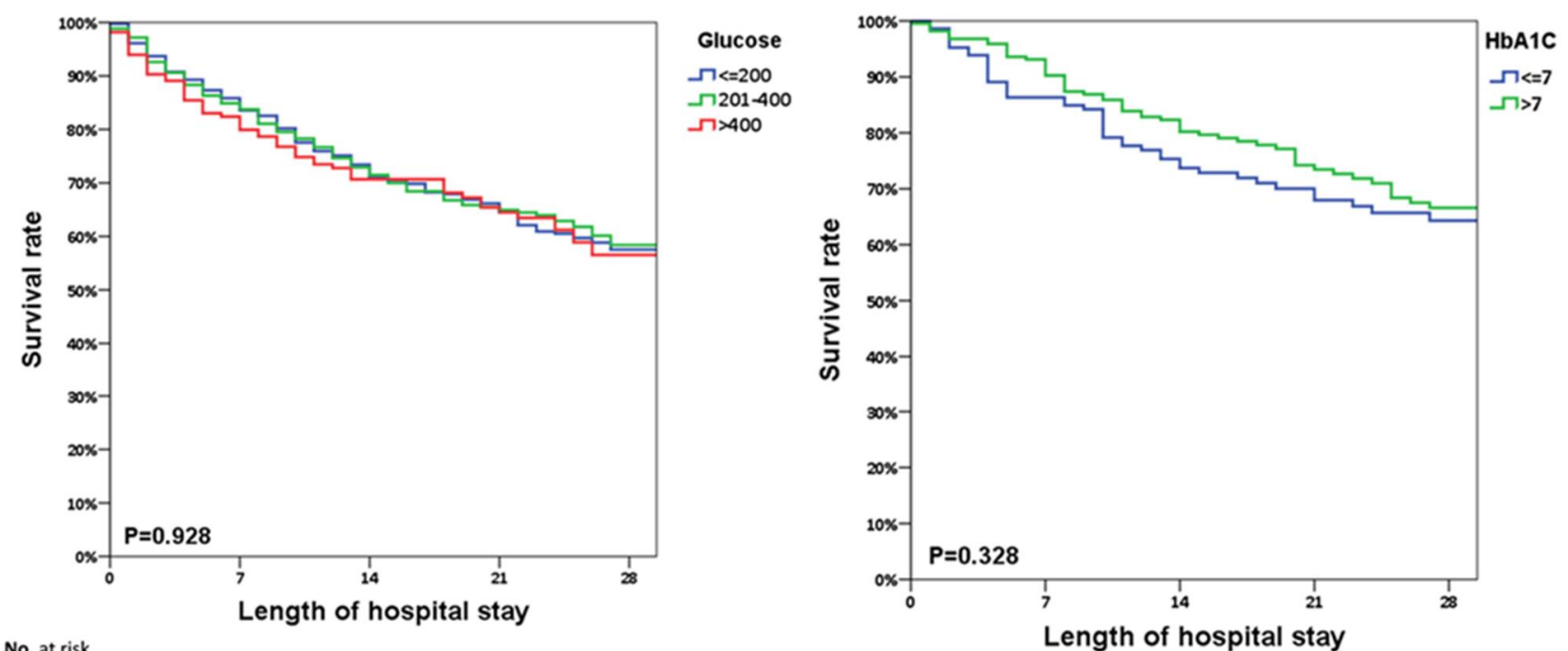

No. at risk

$\begin{array}{cc}\leq 200 & 412 \\ 201-400 & 352\end{array}$

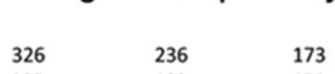

$\begin{array}{rrrr}326 & 236 & 173 & 122 \\ 282 & 189 & 132 & 90 \\ 128 & 94 & 64 & 46\end{array}$


Type 2 diabetes and sepsis outcome

1 In the sensitivity analysis, using a wider time period of one month prior to the admission date 2 for HbA1c collection, the hospital mortality during the sepsis course between the type 2 diabetic 3 sepsis patients with $\mathrm{HbA} 1 \mathrm{c} \leq 7$ and $>7 \%$ did not differ significantly (47.2\% versus $44.3 \%, P=0.471$ ) 4 (Supplement Table 12) (Supplement Figure 4).

5 
Type 2 diabetes and sepsis outcome

\section{Discussion}

2 In the current study, we demonstrated that the outcome of type 2 diabetic sepsis patients was mainly determined by the diabetic complication burdens (represented as the aDCSI score). Somewhat surprisingly, neither the recent glucose control (HbA1c) nor the initial glucose level was associated with hospital mortality in the sepsis course. Physicians should not infer the outcome of a diabetic sepsis patient merely via the recent poor glucose control or initial high glucose level; rather, they should consider the diabetic complication burdens.

Donnelly et al. demonstrated that diabetes was associated with an increased risk of hospitalization due to infection diseases. However, diabetes itself and insulin use were not associated with increased 28-day hospital mortality.[31] Dianna et al. demonstrated that diabetic patients had an excess risk of dying from a range of infection diseases.[32] Both studies used a large cohort; however, their conclusions were conflicting. We infer that the difference was induced by the lack of severity classification of diabetic complications. In our current study, we introduced the utilization of aDCSI score and the results showed that the sepsis outcomes of diabetic patients were mainly determined by the complication burdens of diabetes. This inference was supported by the dose-responsive effect in the trend test in our study.

In this study, we found that the OR for hospital mortality elevated as the aDCSI score increased, and presented in a dose-responsive manner. In the type 2 diabetic sepsis patients with an aDCSI score of $\leq 1$ even had a reverse OR for hospital mortality. However, we did not found an obviously shorter disease course of diabetes (from the first diagnosis date of type 2 diabetes to the index hospitalization date) in the patients with an aDCSI score of $\leq 1$ (median duration = 294 days) compared to those with an aDCSI score of $>2$ (median duration $=306$ days). From this result, we can infer that in type 2 diabetic sepsis patients, diabetes-related complication burdens are the major determinant of hospital mortality, but not merely the diabetes itself.

$\mathrm{HbA1c}$ is a widely used marker that reflects the average glucose level within 120 days. It was reported that $\mathrm{HbA1C}$ was a major outcome predictor in diabetic sepsis patients.[21] However, our study results did not support this argument. There are many studies supporting the influence of long-term glycemic control on diabetic complication development.[33, 34] Long-term poor glycemic control makes diabetic patients prone to infection diseases because of their impaired immune functions.[31] However, the hospital outcome of diabetic sepsis patients presenting with higher $\mathrm{HbA1c}$ may be not as poor as we initially thought because these patients may receive more aggressive blood sugar control in the initial stage of sepsis with insulin.

Hyperglycemia frequently occurs in sepsis patients as a stress response by stimulating 
Type 2 diabetes and sepsis outcome

1 gluconeogenesis, which uses recycled pyruvate and lactate.[35-37] Hyperglycemia may have 2 protective effects in patients because high blood glucose levels increase the diffusion gradient in tissues with abnormal microvasculature caused by sepsis. Our study may indirectly support the above argument. The study by van Vught et al. demonstrated that admission hyperglycemia was associated with adverse outcomes in sepsis course, irrespective of the presence of diabetes.[38] However, our study demonstrated that a high blood glucose level at admission was not associated with the hospital outcome.

Our study has the strengths. We examined the association between type 2 diabetes and sepsis outcomes by concomitantly using the representative nationwide database (LHDB and LHID2000) and the hospital-based database from medical centers (details in the appendix) with laboratory data. Using this method, we longitudinally linked the long-term accumulated diabetic complication burdens, $\mathrm{HbA1c}$ (as a representation of recent glucose control), and initial blood glucose levels at admission to describe the complex trajectory of the disease course in these type 2 diabetic sepsis patients. Our study which evaluated the diabetic complication burdens by using aDCSI score rather than simply adjusting for baseline comorbidities was of important innovation in this topic. Also, in this study of the nationwide database, we used the claims data for procedures such as mechanical ventilation, hemodialysis and so on; therefore the accuracy is much better than the use of ICD codes for acute organ dysfunction. Finally, the detailed information such as blood culture results in the hospital-based database provided a richer understanding in the complex interplay between type 2 diabetes and sepsis, rather than simple taxonomy.

This study has limitations. First, although we were able to link the individual patient's medical information between the hospital-based database and the nationwide database to create a convincing longitudinal cohort study. However, due to the increasing conflict of health database utilization in Taiwan, we abandoned this idea to avoid further severe conflicts. Second, HbA1c was usually measured at the physician's discretion and we initially included only one third of the whole type 2 diabetic sepsis patients with data of HbA1c (366 in 1054), that might induce a significant bias. We therefore use a wider time period of one month to substitute three days as the inclusion criteria to examine the effect of HbA1c on sepsis outcome. However, the OR of HbA1c and hospitalization course remained unchanged in using the larger sample size (953 in 1054). 
Type 2 diabetes and sepsis outcome

\section{Conclusion}

2 In type 2 diabetic sepsis patients, diabetes-related complication burdens are the major 3 determinant of hospital mortality, rather than the diabetes itself. Initial blood glucose levels and 4 HbA1c may not be associated with the hospital outcome of sepsis. 
Type 2 diabetes and sepsis outcome

1

2

3

4

5

6

7

8

9

10

11

12

13

14

15

16

17

18

19

20

21

22

23

\section{References}

1. Vincent JL, Marshall JC, Namendys-Silva SA, et al. Assessment of the worldwide burden of critical illness: the intensive care over nations (ICON) audit. The Lancet Respir Med. 2014;2:380-386.

2. Fleischmann C, Scherag A, Adhikari NK, et al. Assessment of Global Incidence and Mortality of Hospital-treated Sepsis. Current Estimates and Limitations. Am J Respir Crit Care Med. 2016;193:259-272.

3. Singer M, Deutschman CS, Seymour CW, et al. The Third International Consensus Definitions for Sepsis and Septic Shock (Sepsis-3). JAMA. 2016;315:801-810.

4. Ou SM, Chu H, Chao PW, et al. Long-Term Mortality and Major Adverse Cardiovascular Events in Sepsis Survivors. A Nationwide Population-based Study. Am J Respir Crit Care Med. 2016;194:209-217.

5. Iwashyna TJ, Netzer G, Langa KM, Cigolle C. Spurious inferences about long-term outcomes: the case of severe sepsis and geriatric conditions. Am J Respir Crit Care Med. 2012;185:835-841.

6. Donnelly JP, Nair S, Griffin R, et al. Association of Diabetes and Insulin Therapy With Risk of Hospitalization for Infection and 28-Day Mortality Risk. Clin Infect Dis. 2017;64:435-442.

7. Falguera M, Pifarre R, Martin A, Sheikh A, Moreno A. Etiology and outcome of community-acquired pneumonia in patients with diabetes mellitus. Chest. 2005;128:3233-3239.

8. Shah BR, Hux JE. Quantifying the risk of infectious diseases for people with diabetes. Diabetes care. 2003;26:510-513.

9. Thomsen RW, Hundborg HH, Lervang HH, Johnsen SP, Schonheyder HC, Sorensen HT. Diabetes mellitus as a risk and prognostic factor for community-acquired bacteremia due to enterobacteria: a 10-year, population-based study among adults. Clin Infect Dis. 2005;40:628-631.

10. Benfield T, Jensen JS, Nordestgaard BG. Influence of diabetes and 
Type 2 diabetes and sepsis outcome

hyperglycaemia on infectious disease hospitalisation and outcome. Diabetologia. 2007;50:549-554.

11. Kornum JB, Thomsen RW, Riis A, Lervang HH, Schonheyder HC, Sorensen HT. Type 2 diabetes and pneumonia outcomes: a population-based cohort study. Diabetes care. 2007;30:2251-2257.

12. Thomsen RW, Hundborg HH, Lervang HH, Johnsen SP, Schonheyder HC, Sorensen HT. Risk of community-acquired pneumococcal bacteremia in patients with diabetes: a population-based case-control study. Diabetes care. 2004;27:1143-1147.

13. Kaplan V, Angus DC, Griffin MF, Clermont G, Scott Watson R, Linde-Zwirble WT. Hospitalized community-acquired pneumonia in the elderly: age- and sex-related patterns of care and outcome in the United States. Am J Respir Crit Care Med. 2002;165:766-772.

14. McAlister FA, Majumdar SR, Blitz S, Rowe BH, Romney J, Marrie TJ. The relation between hyperglycemia and outcomes in 2,471 patients admitted to the hospital with community-acquired pneumonia. Diabetes care. 2005;28:810-815.

15. Tsai $\mathrm{CL}$, Lee $\mathrm{CC}, \mathrm{Ma} \mathrm{MH}$, et al. Impact of diabetes on mortality among patients with community-acquired bacteremia. J Infect. 2007;55:27-33.

16. Vincent JL, Preiser JC, Sprung CL, Moreno R, Sakr Y. Insulin-treated diabetes is not associated with increased mortality in critically ill patients. Crit care. 2010;14:R12.

17. Esper AM, Moss M, Martin GS. The effect of diabetes mellitus on organ dysfunction with sepsis: an epidemiological study. Crit care. 2009;13:R18.

18. Moss M, Guidot DM, Steinberg KP, et al. Diabetic patients have a decreased incidence of acute respiratory distress syndrome. Crit Care Med. 2000;28:2187-2192.

19. Thomsen RW, Hundborg HH, Lervang HH, Johnsen SP, Sorensen HT, Schonheyder HC. Diabetes and outcome of community-acquired pneumococcal bacteremia: a 10-year population-based cohort study. 
Type 2 diabetes and sepsis outcome

Diabetes care. 2004;27:70-76.

20. Graham BB, Keniston A, Gajic O, Trillo Alvarez CA, Medvedev S, Douglas IS. Diabetes mellitus does not adversely affect outcomes from a critical illness. Crit Care Med. 2010;38:16-24.

21. Gornik I, Gornik O, Gasparovic V. HbA1c is outcome predictor in diabetic patients with sepsis. Diabetes Res Clin Pract. 2007;77:120-125.

22. Schuetz $P$, Jones $A E$, Howell $M D$, et al. Diabetes is not associated with increased mortality in emergency department patients with sepsis. Ann Emerg Med. 2011;58:438-444.

23. Wiener RS, Wiener DC, Larson RJ. Benefits and risks of tight glucose control in critically ill adults: a meta-analysis. JAMA. 2008;300:933-944.

24. Yamada T, Shojima N, Noma H, Yamauchi T, Kadowaki T. Glycemic control, mortality, and hypoglycemia in critically ill patients: a systematic review and network meta-analysis of randomized controlled trials. Intensive Care Med. 2017;43:1-15.

25. Chao PW, Shih CJ, Lee YJ, et al. Association of postdischarge rehabilitation with mortality in intensive care unit survivors of sepsis. Am J Respir Crit Care Med. 2014;190:1003-1011.

26. Angus DC, Linde-Zwirble WT, Lidicker J, Clermont G, Carcillo J, Pinsky MR. Epidemiology of severe sepsis in the United States: analysis of incidence, outcome, and associated costs of care. Crit Care Med. 2001;29:1303-1310.

27. Lin CC, Lai MS, Syu CY, Chang SC, Tseng FY. Accuracy of diabetes diagnosis in health insurance claims data in Taiwan. $J$ Formos Med Assoc. 2005;104:157-163.

28. Young BA, Lin E, Von Korff $M$, et al. Diabetes complications severity index and risk of mortality, hospitalization, and healthcare utilization. Am J Manag Care. 2008;14:15-23.

29. Chang HY, Weiner JP, Richards TM, Bleich SN, Segal JB. Validating the adapted Diabetes Complications Severity Index in claims data. Am J Manag Care. 
Type 2 diabetes and sepsis outcome

2012;18:721-726.

30. Chen HL, Hsiao FY. Risk of hospitalization and healthcare cost associated with Diabetes Complication Severity Index in Taiwan's National Health Insurance Research Database. J Diabetes Complications. 2014;28:612-616.

31. Donnelly JP, Nair S, Griffin R, et al. Diabetes and Insulin Therapy are associated with Increased Risk of Hospitalization for Infection but not Mortality: A Longitudinal Cohort Study. Clin Infect Dis. 2016.

32. Magliano DJ, Harding JL, Cohen K, Huxley RR, Davis WA, Shaw JE. Excess Risk of Dying From Infectious Causes in Those With Type 1 and Type 2 Diabetes. Diabetes care. 2015;38:1274-1280.

33. Nathan DM, Genuth S, Lachin J, et al. The effect of intensive treatment of diabetes on the development and progression of long-term complications in insulin-dependent diabetes mellitus. NEJM. 1993;329:977-986.

34. Intensive blood-glucose control with sulphonylureas or insulin compared with conventional treatment and risk of complications in patients with type 2 diabetes (UKPDS 33). UK Prospective Diabetes Study (UKPDS) Group. Lancet. 1998;352:837-853.

35. Marik PE, Bellomo R. Stress hyperglycemia: an essential survival response! Crit Care. 2013;17:305.

36. Dungan KM, Braithwaite SS, Preiser JC. Stress hyperglycaemia. Lancet. 2009;373:1798-1807.

37. Langouche L, Van den Berghe G. Glucose metabolism and insulin therapy. Crit Care Clin. 2006;22:119-129, vii.

38. van Vught LA, Wiewel MA, Klein Klouwenberg PM, et al. Admission Hyperglycemia in Critically III Sepsis Patients: Association With Outcome and Host Response. Crit Care Med. 2016;44:1338-1346. 
Type 2 diabetes and sepsis outcome

\section{Figure Legends}

2 Figure 1 Logistic regression analysis analyzing the odds ratios of hospital mortality in

3 sepsis patients with type 2 diabetes and different diabetic complication burdens 4 (aDCSI scores).

5 Figure 2A The Kaplan-Meier analysis with log-rank test for hospital course of 6 mortality between the type 2 diabetic and non-diabetic sepsis patients. Figure 2B 7 Comparison of Initial blood glucose levels in the surviving and dead diabetic sepsis 8 patients.

9 Figure 3 Kaplan-Meier analysis with log-rank test for type 2 diabetic sepsis patients 10 with different initial blood glucose levels and HbA1c.

$\underline{\text { Table }}$

13 Table 1 Demographic characteristics, comorbidities, laboratory data, hospital course, 14 and outcomes of matched type 2 diabetic and non-diabetic sepsis patients.

\section{Additional Files}

\section{Supplement Figures}

\section{Supplement Figure 1}

19 The participant selection process of combined nationwide and hospital-based

20 databases.

\section{Supplement Figure 2}

22 The participant selection process of the hospital-based database.

\section{Supplement Figure 3}

24 The stratification analysis by age (every 10 years) according to dichotomized aDCSI 25 scores (0-2 and $\geq 3$ ). 
Type 2 diabetes and sepsis outcome

\section{Supplement Figure 4}

2 Kaplan-Meier analysis with log-rank test to determine the difference of survival rate

3 in $\mathrm{HbA} 1 \mathrm{c} \leq 7 \mathrm{and}>7$ by using a wider collection time period of one month prior to the

4 admission date.

5 Supplement Tables

6 Supplement Table 1

7 Classification of infection sites by ICD-9-CM codes

8 Supplement Table 2

$9 \quad$ ATC drug codes

Supplement Table 3

11 Procedure codes in the national health insurance database

12 Supplement Table 4

13 adapted Diabetes Complications Severity Index score (aDCSI score) by ICD-9-CM 14 Codes

15 Supplement Table 5

16 Nationwide database: demographic characteristics, comorbidities, and medications

17 in type 2 diabetic and non-diabetic sepsis patients before and after propensity score 18 matching

\section{Supplement Table 6}

20 Nationwide database: odds ratio of mortality related to type 2 diabetes and its 21 complication severity in different adjusted mode

\section{Supplement Table 7}

23 Hospital-based database: odds ratio of type 2 diabetes and baseline comorbidities

24 for sepsis mortality in type 2 diabetic and non-diabetic sepsis patients 
Type 2 diabetes and sepsis outcome

\section{Supplement Table 8}

2 Hospital-based database: demographic characteristics, laboratory data, and hospital

3 course of survived and dead type 2 diabetic sepsis patients

\section{Supplement Table 9}

5 Hospital-based database: odds ratio of demographic characteristics and laboratory

6 data for sepsis mortality in type 2 diabetic sepsis patients

7 Supplement Table 10

8 Hospital-based database: comparison of hospital mortality between type 2 diabetic

9 sepsis patients with different $\mathrm{HbA1c}$

\section{Supplement Table 11}

11 Hospital-based database: comparison of hospital mortality between type 2 diabetic 12 sepsis patients with different initial glucose level

\section{Supplement Table 12}

14 Hospital-based database: comparison of hospital mortality between type 2 diabetic 15 sepsis patients with different HbA1c level 
Type 2 diabetes and sepsis outcome

\section{Declarations}

\section{Ethics approval and consent to participate:}

3 This study was approved by two Institutional Review Board :

4 (1) The Institutional Review Board of Taichung Veterans General Hospital (No. CE13233) for the 5 hospital-based database study.

6 (2) The Institutional Review Board of China Medical University (CMUH104-REC2-115) for the 7 nationwide database (LHID2000 and LHDB) study.

\section{Consent for publication:}

$9 \quad$ Not applicable.

\section{Availability of data and material:}

11 The datasets analyzed during the current study are not publicly available because it needed special

12 apply to the national health insurance database of Taiwan.

\section{Competing interests:}

14 None of the authors have any conflicts of interest to disclose. We confirm that we have read the 15 Journal's position on issues involved with unethical publication and affirm that this study is 16 consistent with those guidelines.

\section{Funding:}

18 This work was supported by grants from the Ministry of Health and Welfare, Taiwan 19 (MOHW107-TDU-B-212-123004), China Medical University Hospital, Academia Sinica Stroke 20 Biosignature Project (BM10701010021), MOST Clinical Trial Consortium for Stroke (MOST 21 106-2321-B-039-005).", Tseng-Lien Lin Foundation, Taichung, Taiwan, and Katsuzo and Kiyo 22 Aoshima Memorial Funds, Japan.

23 The funders had no role in the study design, data collection, analysis, decision to publish, or 24 preparation of the manuscript. No additional external funding was received for this study. 
Type 2 diabetes and sepsis outcome

\section{Authors' contributions:}

2 The authors' individual contributions are as follows. Conception and design: Ming-Shun Hsieh, 3 Chorng-Kuang How, Yi-Tzu Lee, and Chen-June Seak. Data analysis and interpretation: Jen-Huai 4 Chiang, Vivian Chia-Rong Hsieh, and Chiann-Yi Hsu. Manuscript writing: Ming-Shun Hsieh. Final 5 approval and critical revision: Pau-Chung Chen, Sung-Yuan Hu, and Sheng-Hsiang Ma. All authors 6 read and approved the final manuscript. (more detail in the submission system)

\section{Acknowledgements:}

8 We thank the Biostatistics Task Force of Taichung Veterans General Hospital, Taichung, Taiwan, 9 Republic of China, for their assistance and advice in the statistical analyses.

10 We also thank the Clinical Informatics Research and Development Center of Taichung Veterans 11 General Hospital, Taichung, Taiwan, Republic of China, for their assistance in data retrieval from 12 the electronic database and further classification.

\section{Appendix}

The hospital-based database was collected from the following three medical centers:

1. Taipei Veterans General Hospital

Ming-Shun Hsieh; Chorng-Kuang How; Yi-Tzu Lee;

2. Taichung Veterans General Hospital:

Sung-Yuan Hu; Chiann-Yi Hsu;

3. Lin-Kou Medical Center, Chang Gung Memorial Hospital: 